\title{
Algorithm to find technical solutions for the modernization of the cold rolling mill of large diameter pipes
}

\author{
Goncharov, KA, Chechulin, Yu.B \\ Institute of Mechanics and Machine Building \\ Yeltsin Ural FederalUniversity \\ Mira street,19- M327, Ekaterinburg \\ RUSSIAN FEDERATION \\ gonkonstant@yandex.ru uchechulin@mail.ru http://www.ustu.ru/en/home
}

\begin{abstract}
The problem of reconstruction and modernization of manufacturing equipment for the production of large diameter pipes was considered in this paper. To assess the strength and the technical resource of the equipment was used mathematical modeling based on the experimental results and consistent solution of nonlinear boundary value problem of the elasticity theory. The results of solution for the tube cold mill, which were used for design of the new equipment, are given. Key-Words:-Stress-state, finite element method, structural non-linearity, non-linear boundary value problem, a cold rolling mill for tubes, roller bearing
\end{abstract}

\section{Introduction}

Development of nano-structured materials and new methods of thermo-mechanical treatment to increase their strength classes, triggers a significant increase in load on the metallurgical process equipment for metal forming. This requires a search for new, alternative design solutions to ensure the reliability of modernized equipment. As you know, one of the major causes of failures of equipment is the local concentration of stress in the contact areas are mutually coupled moving parts. As a result, there is a loss of strength of the individual elements of the design, reducing the accuracy of the mutual movement of its components, adversely affecting the quality of our products.

\section{Problem Formulation}

Under these conditions, reconstruction and design of new machines need to analyze in detail and explaining the reasons that limit the lifespan of existing equipment. The first necessary step of the analysis is the technical measure loads stresses, displacements, temperature conditions, the current characteristics, and the pressure in air hydraulic actuators working equipment in real time. The second important step is the determination of the stress-strain state of interacting units on the basis of established by calculation and experiment actual loading conditions, and the mathematical and physical modeling. It is very effective and low-cost methods for mathematical modeling should include modern methods of numerical analysis, in particular - the finite element method. The results of the preliminary study determine the direction of the search of innovative and original solutions. Thus, the third step is the fundamental change in a dangerous situation, followed by a mathematical test of the stressstrain state of the newly proposed structure. The proposed algorithm is examined on the example of a specific task, which is very relevant in the production of large diameter pipes.

\subsection{The actuator of the cold rolling tube mill}

Seamless cold-deformed pipes, produced by large mills KHPT-250, are used in many industries without further machining. The main feature of the original design of this type of mill is a roller cassette lightweight type with movable supports of the work rolls. Contain movable supports multi-row roller bearings, inner rings mounted on the roll neck and external - in steel thin-walled rings, the outer diameter of which does not exceed the dimensions of the work roll barrel. Roller cassette during rolling reciprocating over the length $1008 \mathrm{~mm}$ with a frequency of 60 cycles per minute. Rings of movable bearings roll along a fixed plate, mounted in a massive stationary frame, which is shown in Figure 1. 


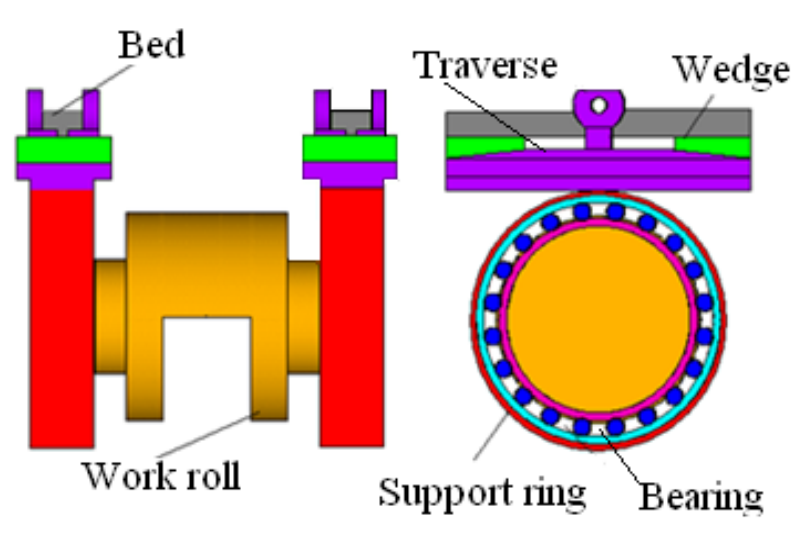

Fig. 1 Structural elements of tube rolling mill KHPT-250

Bearings of working rolls perceive high radial loads that reaches the value $4000 \mathrm{kN}$ on mills KHPT-250. The very limited bearing life demanded an additional analysis of the causes of failures and find new design solutions to improve the efficiency of roller bearings. However, their radial dimensions traditionally limited on the one hand, the distance between work rolls, and the other, - rigidity and strength of the work rolls themselves. Using bearings larger width leads to uneven load distribution between both rows of rollers and the individual rolling elements between each row. This explains the need for ultra-light series bearings with relatively small capacity. The consumption of expensive large-size bearings with an outer diameter of $960 \mathrm{~mm}$ reached 20 units per year to the one tube mill. The analysis of failure of movable bearings mill KHPT-250 showed, that there are chipping on outer ring raceways due to high contact stresses and friction of the rollers relative to the tracks. The destruction of the bearing rings and the separator is due to the axial movement of the bearing rolling elements and pinching. On the destruction process is also influenced by local wear of the rollers until the formation of flats as a result of jamming between the tracks of the rings. The joint dynamic model of the inter-related elements and scheme of its loading was produced for the numerical evaluation of the causes of failures the bearing.

\subsection{Development of a mathematical model}

Consider the contact retaining ring with the traverse on the example of the calculation model, which is shown in Figure 2. A generalized model of the upper work roll includes joint complex elastically deformable parts, which are shown in Figure 2 a. In the left part of this figure is shown the calculation system in original unloaded state, in the right part - in a deformed state under the action of rolling forces. The peculiarity of the computational model is the need for a joint account of volume strain of traverse, wedge, work roll, outer ring of the bearing and the local deformation of the rolling element bearings, which have a direct impact on the uneven load distribution along the rolling elements and between them. It is obviously, that the stiffness of tread ring is much less the stiffness of traverse. Thence for the choice of algorithm of solution of the contact problem with the using of a nonlinear finite element, we can introduce the assumption, that node points of retaining ring are arranged so, that instead of the initial line contact will occur the contact at the contact area, which have a width L. Here the initial values of its dimensions can be determined based on the Hertz formula [1,2]. These assumptions are quite reasonable in relation to the massive bodies, but contact elements are different by the diversity of geometry [3]. In the analyzed case one of the bodies in contact - retaining ring is a shell structure with lowered stiffness, and the second - a solid body with a complex geometry.

Currently, there are a limited number of scientific works, which devoted the contact interaction of a massive body and shell [1,4], all of them are based on the use of numerical methods of solution.

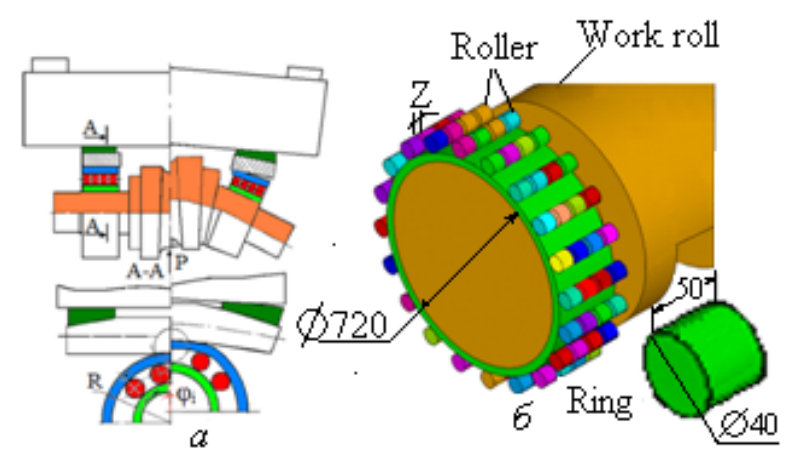

Fig. 2 Creation of the computational model of the camp (a - to the question of development computational model of the actuator of the tube mill, b - to the question of the development of computational models of rolling bearings)

For a reliable calculation of the stress-strain state of roller bearing using a numerical method is necessary to have a mathematical model that takes into account all the factors mentioned above. Structural diagram of four-row rolling bearing which includes in its composition an outer and an inner ring and rollers is shown in Figure 2b. It is seen, that there is a gap $\mathrm{Z}$ between rollers in the longitudinal direction. Single roller bearing and its size is shown in bottom right.

The solution of the contact problem with using FEM is shown by the example of the 
interaction of the inner ring with roller bearing, which is mounted on the shaft neck. When developing a mathematical model is of particular importance the number of nodes in the area of possible contact. To account the effect of ring's stiffening on the loading of roller, the width of the zone of possible contact of was pre-determined by the formula of Hertz and then was increased tripled. It was made for accounting irregularities size of this zone along the length of the roller when choosing a calculation scheme. On the basis of the approach, the pre-test problem has been solved, for which there is an analytic solution. The solution of this task is necessary to assess the effect of the number of nodes of the finite element model on the accuracy of the results of the solution of the contact problem.

The contact of two steel cylinders with a length of $200 \mathrm{~mm}$ and radius $\mathrm{R}_{1}=25 \mathrm{~mm}$ and $\mathrm{R}_{2}=50 \mathrm{~mm}$, which are shown in Figure 3, was considered in this task. It was assumed that the lower large-diameter cylinder is fixed and the upper half of the cylindrical surface of the cylinder with the smaller radius is loaded a uniform pressure, the value of which is equal $\mathbf{p}$. Then the value of the resultant vertical load, which is acting on the contacting cylinders is equal to $\mathrm{F}=\mathbf{p} \cdot \mathrm{D}_{1} \cdot \mathrm{L}$, and the value of $\mathrm{a}$ uniformly distributed linear load, that is used in the formula for calculating the Hertzian contact stress is equal to $q=F / L=p \cdot D_{1}$.

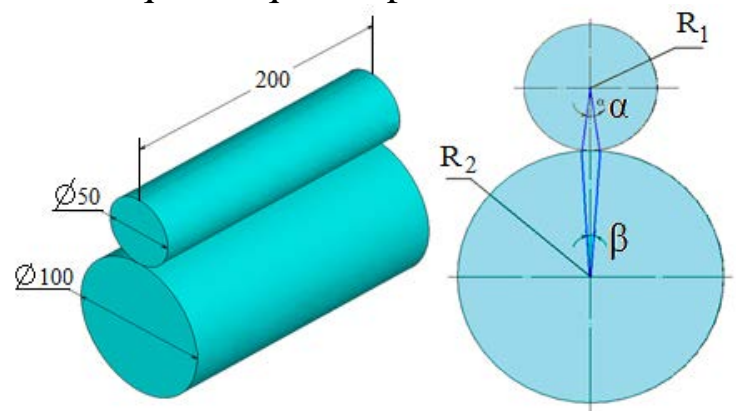

Figure 3 Contact interaction of the two cylinders

In the above formulation the contact problem is structurally non-linear, therefore its solution was carried out by successive approximations with providing pre-specified accuracy of the computational process. Furthermore, the loading step procedure used in the case where, for a given external load iterative process does not converged within the predetermined number of approximations In this case, the load is successively decreased halved until was not ensured convergence of the iterative process. If the number of approximations in the next step of loading was exerted less than 4 , then the value of the load at the next step was increased doubled.

The contact area is a rectangle, when the contact of two cylinders takes place, one side of this rectangle is equal to the length of the contacting bodies and the other side is determined by the Hertz formula

$$
a=\sqrt{q \cdot \frac{R_{1} \cdot R_{2}}{E \cdot\left(R_{1}+R_{2}\right)} \cdot \frac{32 \cdot\left(1-v^{2}\right)}{\pi}}
$$

Central angles $\alpha$ and $\beta$ on the right fragment in Figure 3 determine the size of the contact area in the finite element model and are calculated from the width of the contact area

$$
\alpha=\frac{\mathrm{a}}{\mathrm{R}_{1}}, \quad \beta=\frac{\mathrm{a}}{\mathrm{R}_{2}}
$$

The value of mutual penetration of the cylinders in this case does not depend on the radii of the cylinder and is defined by the relation

$$
\delta=\frac{8 \cdot\left(1-v^{2}\right) \cdot \mathrm{q}}{\pi \cdot \mathrm{E}}
$$

The value of the contact stresses in relation to the plane problem of elasticity theory, is determined on the basis of the ratio, which follows from the classical Hertz contact theory

$$
\sigma_{\mathrm{H}}=0,418 \sqrt{\mathrm{q} \cdot \frac{\mathrm{E} \cdot\left(\mathrm{R}_{1}+\mathrm{R}_{2}\right)}{\left(\mathrm{R}_{1} \cdot \mathrm{R}_{2}\right)}}
$$

When $\mathrm{q}=100 \mathrm{~N} / \mathrm{mm}, \mathrm{E}=200 \mathrm{GPa}, v=0,3$ values, calculated by the formulas (1) - (4) and characterizing the contact of two cylinders with parallel axes, are equal $\mathbf{a}=\mathbf{0 , 2 7 8} \mathbf{~ m m ;} \boldsymbol{\delta}=\mathbf{0 , 0 0 1 1 6}$ $\mathrm{mm} ; \alpha=0,637^{\circ} ; \quad \beta=0,318^{\circ} ; \sigma_{\mathrm{H}}=458 \mathrm{MPa}$.

In developing the calculated finite element model, the width of the contact area was assumed to be $0.85 \mathrm{~mm}$, the number of nodes on the contact area was equal 672. Figure 4 shows a mosaic of the contact stresses in the lower roller and the graph of these stresses along its length.

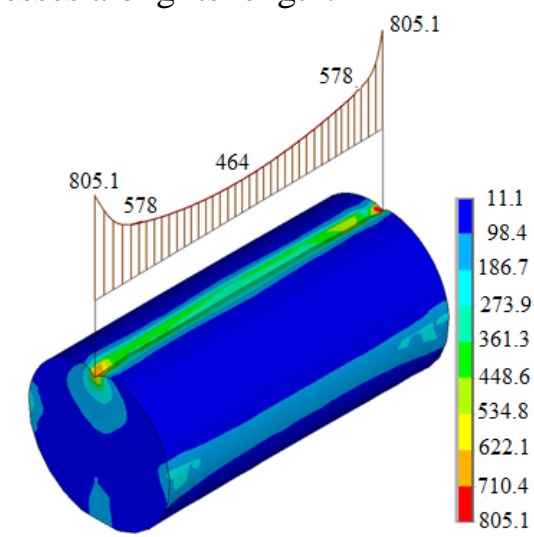

Figure 4 Mosaic of contact stress, MPa 
An analysis of this graph indicates that at the ends of the roller have the distinct maxima of contact stresses. Their value, which is equal 805.1 MPa, significantly, almost twice the above values $\sigma_{\mathrm{H}}=458 \mathrm{M \Pi а}$. Value of the contact stress is equal $464 \mathrm{MPa}$ in the middle of the roller and almost the entire length of its generator. Comparison of this value with the analytical value, obtained by the formula of Hertz (4), indicative of their minor difference. This allows us to draw a conclusion on the admissibility of the selected calculation model for the solution of the contact problem.

The essential difference between the contact stresses at the ends of the roller from the theoretical value due to the fact that the actual cylinders have a finite length. Therefore, the concentration of contact stresses occurs at the ends of roller in the region of the contact area. Mentioned above fact must be considered when choosing the axial profile of the roller bearing [5].

\subsection{Calculation of the stress state of the mill}

The solution for the generalized model, which is shown in Fig. 2, was obtained in view of the considered option to solve contact problems by finite element method. One of the main objectives of the study actuator of mill KHPT-250 is to establish the nature of the stress changes on the rolling element of bearing and the value of maximum stress in the individual elements of the design. A mosaic of the Mises equivalent stresses in the bearing is depicted in Figure 5. The maximum value of analyzed stresses is equal $548.6 \mathrm{MPa}$ and is observed at the edge of the outer bearing ring in the area of contact with the roller.

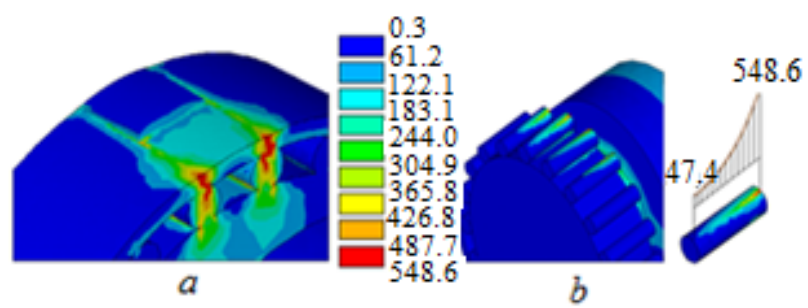

Figure 5 Mosaic of Mises equivalent stresses, MPa: $\boldsymbol{a}$ - in the rolling bearing, $\boldsymbol{b}$ - in the roller of bearing

Distribution of Mises equivalent stress in the bearing rollers is shown in Fig. 5b. It can be seen, that two rollers, which are most loaded, are arranged in close proximity to the vertical plane of symmetry on both sides of it. A graph, describing the distribution of equivalent stresses along the length of the most loaded roller, is depicted to the right of bearing. The graph has a clearly defined nonlinear dependence. The value of the analyzed stress is equal 548.6 MPa at the right end of the roller and on the opposite left end - 47.4 MPa. On all the other rollers of bearing the maximum equivalent stress does not exceed 61.7 MPa.

In the analyzed case of loading the load from the work roll is not transmitted to all four-row rolling element bearing, and only on some of them. In Figure 6 is shown the arrangement of the bearing rollers with numbered rows of rolling elements, which are identified by Roman numerals, and the rolling elements - Arab numerals.

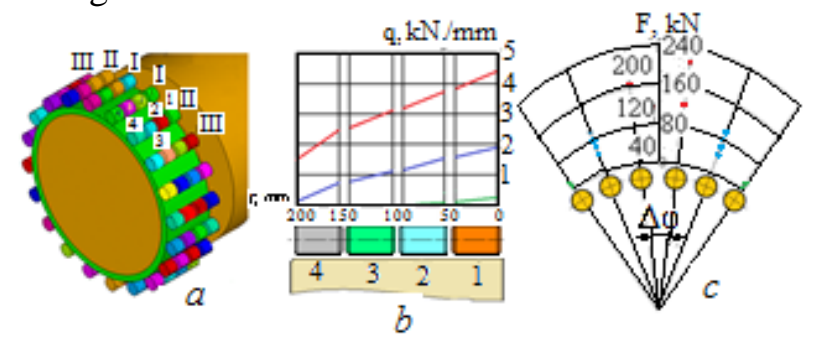

Figure 6 Distribution of the load in the bearing a- the distribution of contact forces on rolling elements of the four-row roller bearing; $b$ - the distribution of the per unit length load on the rolling elements in the axial direction; c - the distribution of the force on rolling elements in the circumferential direction

Analysis of the resulting solution of contact problem shows that in the first and second row are loaded with all four rollers 1,2,3,4. In the third row in contact with the outer and inner ring are only two rollers - 1, 2. Magnitude of the resultant force, acting on a roller, draws special attention to the study, since this force it is necessary to assess the durability of the bearing with the use of traditional methods of calculation. Figure $6 \mathrm{~b}$ shows the distribution of contact forces in rolling element of bearing in the axial direction. Marked in red curve characterizes the distribution of intensity of load per unit length on the rollers of the first row, the blue curve - on the rollers of the second row, the green curve - on the rollers of the third row. The analysis of the curves, which are depicted in Figure 6b, shows a significant decrease in the intensity of the contact load per unit length on the left edge of the roller in comparison with the right edge. For example, value of the intensity of load per unit length on the first roller of the first row is equal to $4.37 \mathrm{kN} / \mathrm{mm}$ on the right edge, and on the left edge analogical value is equal $3.82 \mathrm{kN} / \mathrm{mm}$. The difference between the analyzed load on the right and left edge of the roller is $13.6 \%$. For the fourth roller of the first row of a similar difference between the intensity of the analyzed load is significantly higher - at $37.4 \%$. A similar difference is observed in the rollers bearing of the second row. And in the 
third row the difference between the value of intensity of the load per unit length on the left and right edges of the rollers is less pronounced at $7.2 \%$ for the first roller, and $4.6 \%$ - for the second roller. Fig. 6 c shows the force distribution, which was calculated on the basis of the relation (5) for bearing rolling elements in the circumferential direction.

$$
\mathrm{P}_{\mathrm{T} . \mathrm{K}}=\int_{\mathrm{A}_{\mathrm{n}}} \sigma_{\mathrm{n}} \mathrm{dA}_{\mathrm{n}}+\sum_{\mathrm{j}=1}^{\mathrm{K}} \mathrm{P}_{\mathrm{iH}}
$$

where $\sigma_{\mathrm{n}}$ - stress, which is acting in the direction normal to the contact surface; An - projection of the contact surface on the plane perpendicular to the vector $\sigma_{\mathrm{n}} ; \mathrm{K}$ - the number of structurally nonlinear elements within contact zone of the single roller; $\mathrm{P}_{\mathrm{iH}}$ - the force in the constructive non-linear element, located in the contact area, its value allows to determine the value of the second term in equation (5). The first term in this expression with sufficient precision is determined from equation (6).

$$
\int_{A_{n}} \sigma_{n} d_{A}=\sum_{j=1}^{n}\left(\frac{\sigma_{j}+\sigma_{j+1}}{2}\right) \cdot \Delta l \cdot R_{p} \cdot \sin \Delta \varphi
$$

where Rp -radius of the roller; $\Delta \mathrm{l}$ - distance between adjacent sections, in which all nodes of the finite element model are located; $\sigma_{j}$ - the value of stress in the $\mathrm{j}$ - th section along the length of the roller; $\Delta \varphi$ the value of the central angle between adjacent rows of bearing; $n$ - number of sections on the length of the roller.

Experimental results characterizing the strain distribution across the width of the bearing ring, have been received with using wire gages with the length equal $5 \mathrm{~mm}$ of an alloy of $\mathrm{Cu}-\mathrm{Ni}$. Ten of these strain gauges are glued to outer ring of the roller bearing along of it outer surface at a small distance from the contact area. Compensating strain gage was glued on a fixed frame of the mill. Calibration of the strain gauges was carried out by applying a load to the surface of the work roll groove. The value of the calibration's force was determined with using pressure sensors, which were installed in the hydraulic system. Readings of strain gages yielded experimental stress distribution across the width of the outer ring of the bearing. Direct comparison of stresses with those, which is shown in Fig. 5, is impossible for two reasons:

- strain gages are located not in the contact area, and in the neighboring area, located a short distance from the analysed area;

- strain gages let to record the strain, which may be compressive or tensile. Then, using Hooke's law for plane stress-strain state, we can calculate the stress at the locations of the strain gauges. But in Fig. 5 is shown a mosaic of equivalent stresses, which are used in assessing the strength of the design under complex loading. Graph showing the distribution of the load per unit length to the width of the ring and got on the testimony of strain gages is similar in form to the same graph in Figure $6 b$. This indirect comparison of theoretical and experimental results indicates on the acceptability of the calculational model and the reliability of results of calculations.

For analyzed bearing the ratio of the outside diameter to the internal diameter is equal 1.8 , the durability factor, calculated for this ratio is equal $\mathrm{K}=12.43$. The durability factor, defined by the traditional formula [6] for the case of the placement of bearing in the bore of the fixed hard pillows, is almost 2.5 times lower. The comparison of the law the load change on the rolling elements with the experimental dependence obtained for the bearings from the optically active material [7] is evidenced about of their consistency.

The proposed method of calculating the stressstrain state of the bearing with the joint deformations of individual assemblies of complex systems lets to determine the variation of the load on the rolling elements and along a generator of movable support, not only for the given type of bearing, but for all the bearing assemblies.

\section{Development of a new actuator to increase the resource of the mill for cold rolling tubes}

Based on the calculation results given above, is shown, that the weakest link in the analyzed mechanical system is a rolling bearing.

Change of the stiffness of movable support not gives the desired result, but leads only to a relatively small increase in durability. Therefore, the basic idea of the upgraded version of the design of the actuator mill KHPT-250 was contained in the exclusion of rolling bearings from the system and the replacing of them by additional slide with two parallel support rollers. These rollers were placed between the neck of the working roll and the support rail and had an increased diameter relative to the rolling bodies. The design of the carriage allows it free to self-install under the influence of the contact load. Moreover, is provided uniform pressure distribution between the bearing rollers of each pair. This important fact lets to eliminate undesirable effect of shifting the axis of a support roll relative to the normal to the support surface of rail at reverse motion cassette. Mentioned fact contributes to additional improve the accuracy of rolled tubes $[8,9]$. 
The new design of the working mill stand KHPT-250 is shown in Fig. 7.

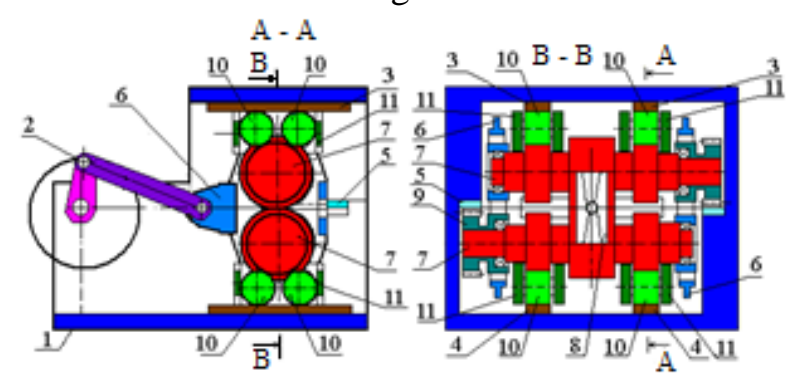

Figure 7 The improved actuator of the working stand of the mill HPT-250

The working stand includes a unmovable frame 1 , which established a crank drive 2. Two top support rail, two lower support rail and two racks 5, intended for the drive of the rotation of the work rolls, are secured in a fixed frame. Cartridge with two work rolls 7 is attached to the crank drive. In the central part of each work roll is mounted calibers 8 . On both sides of the caliber on the work roll there are supporting surfaces, and on the it edge is located gear 9, which is meshed with the rack. During the technological cycle, the crank mechanism moves the cassette with the work rolls. Through meshing gear, mounted on the edge of the working roll, with the rack, the rotational movement is reported on work rolls. As a result, the rolling of tube billet is produced with use of calibers. The load, required to deform the tube blank, transfers through the caliber on the support surfaces of the supporting rolls and further on the supporting traverse 3 and 4 . Carriage 11, which is not associated directly with the cassette 6 , enables for support rollers to occupy the position of equilibrium between the rail and the support surface of the work roll.

A distinctive feature of the new version is the removal of rolling bearings from equipment, which is loaded the full rolling force through the use of an additional carriage. The carriage comprises a parallel twin-mounted supporting rollers, which are arranged between the neck of the working roll and the support surface of rail.

In this case, the design of the carriage provides a free choice of its location under the action of contact pressure between supporting plates and contacting with them cylindrical surfaces of the rolls. This provides a uniform pressure distribution between the bearing rollers of each pair. The marked negative effect takes place in the working stand like "quarto". At the same time is eliminated negative effect of shifting the axis of a support roll relative to the normal to the reference surface of the rail. The proposed design scheme provides an additional increase in the dimensional accuracy of rolled tubes.

\section{Conclusion}

Based on the completed research of the current version of the actuator of the mill KHPT-250 was found, that the four-row rolling bearing is the weak link of mechanical system. The bending of work roll is accompanied by an extremely uneven distribution of external load on rolling elements. Rollers of extreme row, faced to the work roll, are subjected to action of load, twice surpassing similar load for the opposite extreme row. In the circumferential direction the maximum load is acted on the rollers, located at the shortest distance from the vertical axis of symmetry. Depending on the stiffness of the outer ring of bearing, are loaded from four to eight rollers The marked features do not allow to increase the technical resource of the mill.

A new version of the actuator was designed and tested for operation of mills KHPT-250. Numerous experimental and statistical results with the use of strain gauges and pressure sensors were obtained after the introduction of the upgraded drive of mechanism. Furthermore, a plurality of measurements of thicknesses of pipe at different cross-sections along its length was held. The accumulated results of experiments and measurements, and the practice of exploiting of the mill clearly showed the validity of the proposed structural solution. This new design of the actuator allowed to increase repeatedly the duration of work of the mill and improve the accuracy of the pipes. Replacing of the multi-row roller bearing selfsetting mobile carriage with two support rollers lets to exclude the local loading on the outer ring from high enough effort rolling. Using two support rolls instead of a rolling bearing is allowed to reduce in three time the value of deflection of rail in comparison from the original design and thus increase the accuracy of the rolled tube. Application of the new actuator in the mill of the KHPT-250 is allowed for three years, more than 20 times to reduce the cost of maintaining its operation. Furthermore the wear resistance of baseplate was increased repeatedly, the field deviation of the mean thickness of wall of the rolled pipe is decreased down at $60 \%$.

References:

[1] Kim J. H. A new computational approach to contact mechanics using variable-node finite elements. International Journal for Numerical Methods in Engineering. 2007, Vol. 73, Iss. 13, P. 1966-1988. 
[2] Garcia de Jalon J. Kinematic and dynamic simulation of multibody systems. The real-time challenge.New-York: Springer-Verlag, 1994. 449 p. [3] Novikov S.P., Ivchenko V.N., Podoprigora Y., Sakalo V.I. Contact a massive body with a cylindrical shell. Dynamics and strength of transport vehicles. Bryansk, 2000. P. 72 - 78.

[4] Kalker J. J. Three-dimensional elastic bodies in rolling contact. London: Kluwer Academic Publisher, $1990.314 \mathrm{p}$.

[5] Johnson K. Mechanics of contact interaction. M: Mir, 1989. 509 p.

[6] Levina Z.M., Reshetov D.N. Contact stiffness machines. - M.: Mashinostroyeniye, 1971. 264p.
[7] Grudev A.P. The theory of rolling. M: Metallurgiya, 1988. $240 \mathrm{p}$.

[8] Goncharov KA, Chechulin Yu.B. The stressstrain state of the details of the construction of coldrolling tube mill. Bulletin of the Nizhny Novgorod University. N. Novgorod, 2011. Number 4. Part 4. P.1451-1453.

[9] Pat. Number 21413888 Russian Federation, IPC B21D5/10. Working stand of the cold rolling tube mill / Yu.B Chechulin, A.M Cauzov, A.A Fedorov, G.A Novenko, V.K Khristenko, A.M Sevostyanov, B.Ya Sokolinsky. (Russian Federation). - Publ. B.I number 32, 1999

\section{Creative Commons Attribution License 4.0 (Attribution 4.0 International, CC BY 4.0)}

This article is published under the terms of the Creative Commons Attribution License 4.0

https://creativecommons.org/licenses/by/4.0/deed.en_US 